\title{
Prendre en compte la continuité historique pour penser le politique aujourd'hui
}

Taking Account of Historical Continuity in Addressing the Political Today 論當代中國政治思想三大傳統的歷史連續性

\section{Yang Gan}

Traducteur : Sébastien Billioud

\section{(2) OpenEdition}

\section{Journals}

Édition électronique

URL : http://journals.openedition.org/extremeorient/137

DOI : 10.4000/extremeorient. 137

ISSN : 2108-7105

Éditeur

Presses universitaires de Vincennes

\section{Édition imprimée}

Date de publication : 1 octobre 2009

Pagination : 125-139

ISBN : 978-2-84292-237-5

ISSN : 0754-5010

\section{Référence électronique}

Yang Gan, «Prendre en compte la continuité historique pour penser le politique aujourd'hui », ExtrêmeOrient Extrême-Occident [En ligne], 31 | 2009, mis en ligne le 01 octobre 2012, consulté le 01 mai 2019. URL : http://journals.openedition.org/extremeorient/137 ; DOI : 10.4000/extremeorient.137 


\title{
Prendre en compte la continuité historique pour penser le politique aujourd'hui
}

\author{
Gan Yang ${ }^{1}$ \\ Traduction du chinois de Sébastien Billioud
}

La République populaire de Chine fête cette année ses soixante ans. Prendre pour référence l'ensemble de ce processus historique me semble aujourd'hui nécessaire pour repenser la question de la réforme chinoise depuis 1979. Il ne convient pas de limiter notre perspective sur les réformes en ne prenant en compte que les trente dernières années, comme on le fait habituellement. En effet, procéder ainsi rompt non seulement la continuité historique entre les trente premières (1949-1979) et les trente dernières années (1979-2009) de la nouvelle Chine, mais conduit presque inévitablement à opposer complètement ces deux périodes. Nombreux sont ceux qui, soulignant le succès éclatant de la réforme économique chinoise, présupposent que la période qui la précède est à rejeter en bloc, ce qui incite à penser que seul ce rejet permet d'expliquer les prouesses ultérieures. Mais on entend aussi depuis quelque temps un autre type de discours qui consiste à faire référence à la période maoïste pour critiquer les problèmes actuels et nier la valeur des réformes de l'ère ouverte par Deng Xiaoping. En d'autres termes, on utilise la première période de la nouvelle Chine pour attaquer la seconde. En tout état de cause, les débats de tous ordres sur les réformes qui ont eu lieu, ces dernières années, au sein même de la société chinoise, ont eu pour mérite de poser clairement la question de la continuité historique dans la Chine nouvelle. En conséquence, nous prenons conscience de la nécessité de trouver un nouveau discours et une perspective plus englobante

1. Ce texte est une version modifiée d'un article paru à l'origine à Pékin dans le numéro 2007/6 de la revue Lire (Dushu) sous le titre: «La Voie chinoise, trente ans et soixante ans» (Zhongguo daolu, sanshi nian yu liushi nian). Il a suscité de nombreux débats et polémiques. Certaines parties du texte présenté ici ont été largement adaptées par le traducteur en accord avec l'auteur afin de mieux répondre au thème de ce numéro d'Extrême-Orient Extrême-Occident. 
pour rendre compte de l'ensemble de l'histoire de la République durant ces soixante dernières années.

\section{La formation d'un consensus sur la suite de la politique de réformes}

Il me semble que, quelle que soit la perspective dans laquelle on se place, tout discours opposant les trente années de la période maoïste et les trente années suivantes manque quelque chose. En effet, il devient alors difficile de véritablement expliquer la totalité du processus historique à l'œuvre au cours de ces soixante années de la République. Avant de développer cette idée, il me faut insister sur deux éléments : d'une part, en dépit de tous les problèmes sociaux et les fractures de la société chinoise actuelle, les résultats obtenus par la politique de réforme sont sans précédent. Je pense aux quatre cents millions de personnes sorties de la pauvreté depuis près de vingt-cinq ans. D'autre part, même si la situation des ouvriers et des paysans chinois est parfois encore problématique on ne peut, sans déformer la réalité, assimiler leur vie à un enfer. En somme, depuis l'ouverture, la situation d'une très grosse majorité de la population s'est considérablement améliorée et l'éternel problème des famines récurrentes a enfin été réglé. Ce sont des faits.

Il ne fait aucun doute que les réformes conduites à partir des années 1990 ont généré des écarts croissants de revenus, de sorte que le problème de la justice sociale est devenu aujourd'hui une préoccupation première. Pourtant, tous les débats et polémiques de ces dernières années sur les réformes ne peuvent être caricaturés comme reflétant «la fin d'un consensus». Bien au contraire, ce à quoi nous assistons, c'est plutôt à la formation d'un «nouveau consensus sur les réformes ». Ce consensus exige aujourd'hui la justice sociale plutôt que la «productivité à tout prix », un bien-être matériel partagé plutôt que «l'enrichissement préalable d'une minorité » et un fondamental «souci des gens » (yi ren wei ben) plutôt que la quête aveugle de la croissance du PIB. Ce consensus nouveau est déjà devenu en Chine une opinion commune majoritaire et a conduit ces dernières années le gouvernement et le Parti à ajuster et réorienter le programme de réformes. Cela s'est traduit par l'élaboration de principes fondamentaux en vue de la construction d'une «société d'harmonie». L'esprit de ce projet est souligné dans les «Propositions du $11^{\mathrm{e}}$ plan quinquennal pour l'élaboration de l'économie et du développement social» approuvées lors de la séance plénière du $16^{\mathrm{e}}$ congrès du PCC. La réforme doit «se soucier davantage de justice sociale et faire en sorte que l'ensemble de la population bénéficie des fruits du développement». Certes, toutes sortes de groupes d'intérêts chercheront sans doute à saboter ce nouveau consensus mais ce qu'il faut souligner, c'est que 
Prendre en compte la continuité historique pour penser le politique aujourd'hui

bien rares sont ceux qui osent s'y opposer frontalement et à visage découvert. En d'autres termes, son autorité est telle qu'on ne peut le contrecarrer facilement et il a au moins pour mérite de servir à restreindre la marge d'action des divers groupes d'intérêts.

\section{Le nouveau consensus et les trois traditions à l'œuvre en Chine contemporaine}

Il me semble que la formation progressive de ce nouveau consensus résulte en réalité de toutes sortes de synthèses opérées à travers ces soixante ans d'histoire de la République. Ce consensus contribue à dissoudre certaines tensions entre la politique menée pendant l'ère maoïste (les trente premières années) et celle qui caractérise la phase suivante (les trente années d'ouverture). Si «l'ancien consensus » (jiu gaige gongshi), qui régnait encore il y a peu, tendait à nier l'apport des trente premières années, le «nouveau » contribue, pour sa part, à le prendre pleinement en compte. À vrai dire, ce «nouveau consensus sur les réformes» est le fruit de trois sortes de traditions qui se font mutuellement écho.

Quelles sont ces trois traditions? La première trouve sa source dans les vingt-neuf années de réforme. Cette période, bien que courte, a vu bon nombre de nouvelles idées, accompagnées d'un nouveau vocabulaire, entrer dans les mentalités et dans la vie quotidienne des Chinois. Cette tradition a pour cœur l'idée de «marché» et comprend bon nombre de concepts avec lesquels nous sommes désormais très familiers comme ceux de liberté, de droits, etc. La seconde tradition est la tradition maoïste, inaugurée avec l'instauration de la République populaire. C'est une tradition qui met l'accent à la fois sur l'égalité et sur la justice. Avec le recul, nous pouvons désormais voir de façon claire que cette tradition maoïste a fait un retour sur scène à partir du milieu et de la fin des années 1990. En effet, à partir de cette période, nombreuses ont été les discussions sur la période maoïste, et à la fin de la décennie, la tradition égalitaire a regagné en vigueur. Une telle situation était certes difficile à imaginer il y a encore dix ans, mais il est désormais indéniable que cette tradition fait de nouveau aujourd'hui partie de notre vie. En dernier lieu, la troisième tradition est celle de la civilisation chinoise façonnée par les siècles, ce que l'on appelle souvent la culture traditionnelle chinoise ou la tradition confucéenne. Pour dire les choses simplement, elle s'exprime principalement dans la vie quotidienne des Chinois à travers la valorisation de relations interpersonnelles (renqing), un attachement à des traditions locales (xiangqing) ou l'importance accordée aux relations familiales. Ce phénomène transparaît clairement à travers les nombreuses séries télévisées diffusées en Chine, notamment celles qui traitent 
de la vie familiale ou de la morale quotidienne et des affaires de mariages ou de divorces.

La coexistence de ces trois traditions est une situation très singulière caractérisant la société chinoise, et plus particulièrement le continent. Si l'on prend Hong Kong comme point de comparaison, la société de ce territoire hérite certes de deux de ces traditions (liberté et marché d'une part, valorisation des relations interpersonnelles et des traditions locales d'autre part), mais non pas de la troisième, c'est-à-dire d'une forte aspiration à l'égalité. Dès lors, la société hongkongaise demeure inégale au plus haut point et si certains s'attellent à améliorer la situation, ceci ne suscite pas pour autant de débat idéologique animé. Si l'on change de perspective pour opérer une comparaison avec les États-Unis, on voit qu'il y existe deux très fortes traditions de liberté et d'égalité, si bien que la tension entre ces deux pôles peut être décrite comme vraiment caractéristique du pays. Mais il y manque cette valorisation des relations humaines et l'attachement au local et, plus encore, la mentalité et la tradition culturelles qui en constituent le substrat.

\section{La voie chinoise : une république socialiste confucéenne}

La formation d'un consensus sur la réforme en Chine ne peut se faire sans prendre en compte les «conditions spécifiques» (guoqing) de la société chinoise et la coexistence de ces trois traditions. Plus encore, je crois que la spécificité d'un nouveau consensus tient au fait qu'il ne peut faire l'économie d'aucune de ces trois traditions : il doit reconnaître la légitimité de chacune d'entre elles et leur permettre à la fois de se contrôler et de se compléter mutuellement.

On peut affirmer que le «consensus actuel sur la réforme» reflète bien, dans sa phase initiale, le recours conjoint à ces trois traditions. En premier lieu, il ne fait aucun doute que le concept même de «société d'harmonie » s'enracine dans la tradition confucéenne et diffère fondamentalement de ce concept occidental de «lutte des classes » auquel le Parti a fait longtemps référence. De plus, l'objectif essentiel de la constitution d'une société d'harmonie est aussi la «prospérité de tous », une aspiration centrale de la tradition socialiste maoïste. Mais, enfin, une telle prospérité ne pourra être atteinte contre le marché et devra l'être à travers l'amélioration progressive des mécanismes de marché mis en œuvre avec la politique de réformes. Nul besoin de préciser que l'alliance de ces trois traditions sera un processus rempli de tensions, de contradictions, voire de conflits ; mais cela n'est pas une mauvaise chose, bien au contraire. Seule la domination d'une tradition écrasant les autres est nuisible. Ce que nous devons précisément combattre, c'est tout mode de pensée exclusif ou de configuration politique qui prenne la forme d'un «jeu à somme nulle». Encore une fois, on peut affirmer 
Prendre en compte la continuité historique pour penser le politique aujourd'hui

que, quelles que soient les tensions à surmonter, le nouveau consensus doit prendre en compte les trois traditions sans en exclure aucune.

Certains critiqueront et diront que de tels propos tendent à nier la lutte des classes. Nous nous trouvons sans doute aujourd'hui devant la nécessité de repenser les distinctions jadis opérées par Mao entre «les contradictions au sein du peuple» (renmin neibu maodun) et «les contradictions entre l'ennemiet nous» (di wo maodun). Dans le processus de réformes en Chine subsistent encore maintes contradictions, tensions et conflits qui, pour la plupart, relèvent de «contradictions au sein du peuple » et ne peuvent être considérés à la légère comme relevant de contradictions avec l'ennemi, caractérisées par une lutte à mort. Nous devons nous opposer à toute personne qui s'abriterait derrière «le caractère du politique » et à toute intention de radicaliser les tensions existant au sein du peuple. Mais en même temps, face à tous ceux qui tendent aujourd'hui à avoir recours, pour aborder les problèmes, à toutes sortes de théories occidentales sur les «genres, les minorités ou les classes», nous devons insister sur le fait que les contradictions entre genres, orientations sexuelles ou groupes ethniques relèvent toutes de «contradictions au sein du peuple» et peuvent donc être résolues ou apaisées. Toute approche visant à élargir le champ de ces contradictions pour les transformer en une opposition insurmontable entre soi et l'ennemi fait fausse route et ne peut conduire qu'à des catastrophes politiques. Nous devons examiner, de façon critique, toutes ces théories occidentales à la mode, qu'elles soient de droite ou de gauche, et ne pas les adopter en bloc, sans réflexion. En réalité, cela fait bien longtemps qu'il n'y a «plus rien de nouveau sous le soleil» et je ne pense pas qu'elles puissent avoir grande valeur pour nous. Les Chinois doivent utiliser leur tête pour penser, leurs propres pieds pour avancer.

À long terme, le consensus sur les réformes qui se forme actuellement en Chine, s'il génère un développement sain, pourra peut-être graduellement manifester le caractère vraiment propre de «la Voie chinoise» (Zhongguo daolu). L'ultime but poursuivi par la réforme chinoise n'est pas de forger un capitalisme à l'américaine, mais d'atteindre ce que j'appellerai une «République socialiste confucéenne» (rujia shehuizhuyi gongheguo). Il y a quelque temps, j'avais déjà avancé que l'idée même de République populaire de Chine (Zhonghua renmin gongheguo) correspondait en fait à celle d'une république socialiste confucéenne. Les raisons en sont les suivantes. Tout d'abord, le sens même du terme zhonghua ${ }^{2}$ fait référence à la civilisation chinoise qui, axée avant tout sur le confucianisme, en vient aussi à assimiler le taoïsme, le bouddhisme et d'autres éléments culturels. En second lieu, l'idée même de «république populaire»

2. Terme qui désigne la «nation chinoise» depuis l'ère républicaine et qui correspond à «de Chine» dans le nom officiel de «République populaire de Chine». N.d.T. 
montre que cette république ne se veut pas capitaliste. Elle englobe l'ensemble du peuple, elle est celle des ouvriers, des paysans et des autres travailleurs ou, pour dire les choses autrement, elle est socialiste. Substantiellement donc, la RPC est une République socialiste confucéenne. Le sens le plus profond de la réforme chinoise est bien là : il s'agit d'approfondir et de développer le contenu d'une telle république, et tel est bien pour nous l'enjeu du XXI ${ }^{\mathrm{e}}$ siècle.

\section{Repenser les soixante ans d'histoire de la République}

En partant des perspectives proposées plus haut, j'ai emprunté ces dernières années une expression à l'ancienne tradition confucéenne du Gongyang pour dire que la réforme, en Chine, doit entrer dans une nouvelle ère caractérisée par « un continuum entre les trois traditions » (tong san tong). Ce qu'il faut souligner, c'est que les traditions de Confucius, de Mao Zedong et de Deng Xiaoping renvoient à une continuité, celle de la civilisation et de l'histoire chinoises. Dès lors, nous devons réexaminer les succès de la réforme en l'inscrivant dans une relation de continuité avec l'ère maoïste. De surcroît, nous devons aussi prendre la mesure du caractère fondateur de la civilisation et de l'histoire traditionnelles chinoises pour la Chine moderne.

Dans la suite de cet article, nécessairement limitée, je vais évoquer la question du lien entre la tradition historique chinoise et la Chine contemporaine et commencer par soulever le problème de la relation entre les trente premières années et les trente dernières de la République populaire.

En premier lieu, il existe évidemment des différences fondamentales entre les périodes maoïste et denguiste. Avec Deng, on passe délibérément du primat de la lutte des classes à la construction de l'économie. Néanmoins, une telle évolution n'aurait pas été, en elle-même, suffisante pour garantir le succès de la réforme économique. L'URSS et les pays d'Europe de l'Est n'avaientils pas eux aussi très tôt abandonné la lutte des classes pour tout consacrer à l'économie? Mais leurs réformes n'ont pu être couronnées de succès. Pourquoi la Chine a-t-elle ainsi réussi alors que les autres échouaient, jusqu'à assister à une complète dislocation de leurs sociétés? Il faut aujourd'hui revenir sur ces questions. Le succès de la réforme chinoise n'est pas ordinaire, mais à ce jour, il n'a pas encore été véritablement analysé.

Souvenons-nous qu'entre le début des années 1980 et celui des années 1990, bien peu d'Occidentaux, que ce soit dans l'opinion publique ou dans le monde académique, avaient une opinion positive de la réforme économique chinoise. La raison était très simple: on se demandait naturellement comment la Chine pouvait réussir alors que l'URSS et ses satellites d'Europe de l'Est n'y parvenaient pas. Tout le monde pensait que le dénominateur commun à tous ces pays 
Prendre en compte la continuité historique pour penser le politique aujourd'hui

étant un système économique planifié au niveau central, un échec en URSS et en Europe de l'Est ne pouvait dès lors laisser beaucoup d'espoir de succès en Chine. Une telle opinion est parfaitement naturelle. Elle l'est d'autant plus que, si l'on observe l'industrialisation et le processus de modernisation de l'URSS à l'époque, le pays semblait largement en avance sur la Chine. La population paysanne y était moindre qu'en Chine, et le niveau de l'encadrement dans les usines était également bien supérieur à ce que l'on trouvait chez nous (les directeurs d'usines et les managers n'avaient guère reçu en Chine qu'une éducation secondaire alors qu'ils étaient au moins diplômés d'université en Union soviétique). Et si l'on évoque les différentiels de niveau de vie, les écarts sont si flagrants qu'ils ne méritent guère que l'on s'y attarde. Alors que dans la Chine des années 1980 les foyers n'étaient même pas encore équipés de téléphones, les appareils électriques, voire les voitures, étaient déjà entrés en URSS et en Europe de l'Est dans le quotidien des gens. Ces pays, de surcroît, n'avaient connu ni l'équivalent de notre «Grand bond en avant», ni la Révolution culturelle, et ils avaient, bien plus tôt que la Chine, fait leur deuil de la lutte des classes pour s'engager dans la construction de leur économie, tout en bénéficiant d'un système politique plus ouvert. Tous ces paramètres étant pris en compte, à quoi imputer leurs difficultés? Comment se fait-il que la Chine, si arriérée sur tant de plans, ait pourtant largement réussi la réforme de son économie?

Face à de telles questions, peut-être n'y a-t-il en réalité qu'une explication. Il se pourrait que la clé du succès des réformes de Deng soit à rechercher, en filigrane, au sein même de l'époque maoïste, et notamment dans le fait que Mao ait ruiné de façon délibérée les efforts visant, après la proclamation de la RPC, à instituer une économie centralisée et planifiée. On peut emprunter la formule de Schumpeter pour décrire la période maoïste comme un processus de «destruction créatrice». Le Grand bond en avant ou encore la Révolution culturelle ont beaucoup détruit dans la Chine de l'époque, mais de telles destructions revêtaient aussi des aspects créateurs. Fut notamment détruite la possibilité de mettre en œuvre une économie planifiée sur le modèle de celle de l'URSS, mais une telle situation permit du même coup de poser les fondements de la future réforme économique de l'ère de Deng.

\section{Quand la «destruction créatrice» de Mao pose les bases des réformes de Deng}

Les destructions de Mao, pour en rester au domaine économique, sont bien connues. En 1958, l'année du Grand bond en avant, Mao sortit le pouvoir de gestion sur $88 \%$ des usines du domaine de compétence ministériel pour le transférer aux gouvernements locaux à divers échelons. La supervision des 
actifs et des entreprises fut alors transmise aux pouvoirs locaux qui se retrouvèrent, au niveau de la province ou du district, mais aussi à celui du district, en charge d'ensembles industriels. Exigeant que chaque district puisse lui-même développer une industrie, Mao décrivait la situation en disant que «même si les moineaux sont petits, ils possèdent pleinement les organes nécessaires pour survivre». Si, en 1961, le mouvement s'inversa sous l'impulsion de Liu Shaoqi qui confia de nouveau la gestion des usines au pouvoir central, Mao, retrouvant son ascendant après 1964, s'opposa à une telle politique et transféra de nouveau les compétences de gestion aux échelons locaux, prônant alors une politique de «république avec un souverain faible» $(x \text { u jun gonghe })^{3}$. Quand on arriva à la période de la Révolution culturelle, autour de 1968, il n'y avait tout simplement plus de plan économique, ce qui est quand même une chose incroyable. Entre le Grand bond en avant et la Révolution culturelle, Mao avait en réalité ruiné toute tentative d'instituer une économie de plan centralisée.

Les faits que je viens de relater sont largement connus, mais ils sont souvent imputés à la propension destructrice caractérisant Mao. Bien peu de gens ont conscience que les actes de Mao ont pu constituer des bases fondamentales pour la mise en œuvre ultérieure de la réforme économique de Deng Xiaoping. Je voudrais me fonder ici sur les travaux de l'universitaire américaine Susan Shirk pour étayer mon argumentation. En 1993, celle-ci publia un livre, The Political Logic of Economic Reform in China, qui était le résultat d'une décennie d'enquêtes en Chine ${ }^{4}$. De façon fort intéressante, elle mit notamment en exergue le fait que la «logique politique» des réformes économiques de Gorbatchev suivait un modèle occidental qui était en revanche loin de s'appliquer en Chine. Elle s'interrogea à partir de là sur l'échec et le succès des réformes. J'insiste sur le fait que nous sommes en 1993, à une époque où la plupart des spécialistes occidentaux doutaient du succès possible de la réforme chinoise. Or, Shirk évoqua une possibilité différente en soulignant le fait que, contrairement à l'URSS, la réforme avait en Chine été lancée sur les bases d'une «décentralisation» maoïste. Du fait du Grand bond en avant et de la Révolution culturelle, la planification économique centrale n'avait jamais pu réellement être mise en œuvre. Et selon Shirk, sans Mao, la réforme chinoise se serait heurtée aux mêmes échecs qu'en URSS et dans les pays d'Europe de l'Est.

À travers de nombreux exemples, Shirk montre à quel point, avant la politique de réformes, le modèle soviétique, centralisé au plus haut point et articulé autour d'entreprises de grande taille, se différenciait d'un modèle chinois

3. C'est-à-dire avec un centre faible. N.d.T.

4. Shirk Susan, The Political Logic of Economic Reform in China, Berkeley, University of California Press, 1993. 
Prendre en compte la continuité historique pour penser le politique aujourd'hui

caractérisé par un nombre infiniment supérieur d'entreprises plus petites, moins spécialisées et techniquement moins avancées, dont la gestion relevait des échelons administratifs les plus divers ${ }^{5}$. Dès lors, les défis à relever furent bien différents dans les deux pays. En Chine, où la réforme ne portait pas à titre principal sur les entreprises d'État (pour lesquelles les difficultés sont les plus aiguës), un nouveau type de structure - les entreprises des bourgs et des villages (xiang zhen qiye) - permit le développement rapide d'une économie dynamique. Il bénéficia pour cela des acquis de la décentralisation maoïste, comme en bénéficia d'ailleurs plus généralement la politique économique de Deng Xiaoping, axée sur le développement local (zones économiques spéciales, etc.).

À propos de la genèse des réformes, Shirk soulève un point très intéressant. Peu après la Révolution culturelle, alors que Deng Xiaoping ne s'était pas encore emparé du pouvoir, il y eut une tentative de revenir au premier plan quinquennal des années 1950 dont beaucoup se souvenaient comme d'un âge d'or. Ce retour à une planification économique centralisée eut lieu pendant les trois années durant lesquelles Hua Guofeng fut réellement aux commandes (1976-1978). Mais selon Shirk, il n'était plus réellement possible de faire ainsi marche arrière après une telle période de décentralisation et ce fut sans doute pour cette raison que Hua Guofeng fut rapidement destitué.

\section{Les entreprises des bourgs et des villages : de Fei Xiaotong à Mao}

Avec l'arrivée au pouvoir de Deng Xiaoping commença une phase pendant laquelle le centre relâcha son emprise en octroyant pouvoir et droits aux localités et aux entreprises. À l'époque, beaucoup insistaient avant tout sur cette nécessaire prise d'autonomie des entreprises et notamment des entreprises d'État, se fourvoyant ainsi sur le plus important: le développement local. Il faut cependant reconnaître que nul n'avait vraiment prévu le succès des entreprises des bourgs et des villages créées en coopération par les autorités locales et les paysans. Ce qui a rendu possible un tel développement, c'est le Grand Bond en avant de Mao qui, en dépit de son échec, posa, avec les entreprises des communes populaires, les bases de l'essor des entreprises des bourgs et des villages.

5. Shirk montre qu'au moment où l'importance du plan était à son paroxysme, le gouvernement central, en Chine, ne contrôlait que 500 produits contre 5500 en URSS. De plus, alors que toutes les entreprises y étaient contrôlées au niveau central, ce n'était en Chine le cas que de $3 \%$ des entreprises d'État. Enfin, en 1978, il y avait en URSS 40000 entreprises contre près de 340000 en Chine (parmi ces dernières, seules 4000 étaient de grande taille). 
Alors qu'à la fin des années 1930, le grand anthropologue Fei Xiaotong écrivait l'ouvrage qui le rendit célèbre, sur la vie paysanne en Chine ${ }^{6}$, il indiqua que la possibilité d'une modernisation chinoise devait passer par les campagnes tout en réalisant l'impossibilité, à l'époque, d'accomplir cette tâche. Si le rêve de Fei Xiaotong commença dans les années 1980 à se réaliser, c'est grâce à l'action de Mao qui, à partir du Grand bond en avant, permit le développement des campagnes (transports, eau et électricité, jusqu'aux soins médicaux prodigués par les médecins aux pieds nus) et leur industrialisation, posant ainsi les bases du développement ultérieur des entreprises des bourgs et des villages ${ }^{7}$. Naturellement, on pourra dire qu'il aurait mieux fallu emprunter une voie moins chaotique, plus normale, vers la modernisation. Mais ce ne sont que des vœux pieux. En réalité, le chemin vers la réforme n'est pas droit et il faut continuellement s'adapter.

\section{Repenser les trente premières années de la République populaire : la question des orientations privilégiées à Yan'an}

On ne peut désormais plus faire l'économie de la question suivante: pourquoi Mao a-t-il ainsi opiniâtrement et de façon incessante entravé jusqu'à les ruiner tous les efforts entrepris pour mettre en œuvre une économie de plan centralisée? Il me semble qu'il nous faut repenser les trente premières années de la République populaire et le sens d'un certain nombre de concepts qui nous sont extrêmement familiers. Ainsi, dans les années 1950, on commença à évoquer la contradiction entre «le rouge et l'expert» (hong yu zhuan), «les relations entre politique et compétence professionnelle», ou entre «les intellectuels et les masses paysannes et ouvrières », «la soumission de l'expert au profane » ou encore «le contrôle de l'économie par le politique», etc. Toutes ces expressions revêtent une signification sociologique profonde et reflètent des contradictions inhérentes à la société chinoise qui ne le sont pas moins. Ces problèmes constituent encore aujourd'hui les difficultés principales de la société chinoise, même s'ils revêtent une forme nouvelle. Par exemple, très récemment encore, les intellectuels tant de gauche que de droite ont tous insisté sur le fait que la Chine est en

6. Fei Xiaotong, Peasant Life in China, A Field Study of Country Life in the Yangtze Valley, Londres, Kegan Paul, 1939, avec une préface de Bronislaw Malinowski. En chinois, le livre s'intitule Jiang cun jingji (Économie d'un village du bas Yangtze). N.d.T.

7. Il est clair que la modernisation centrée sur le développement local pose aussi de nombreux problèmes (doublons dans le processus de production, pollution, amenuisement des finances centrales, etc.). Mais dans l'ensemble, on ne peut, en se fondant sur les problèmes survenus ultérieurement, nier la contribution des entreprises des bourgs et des villages au développement. 
Prendre en compte la continuité historique pour penser le politique aujourd'hui

train de créer une opposition entre une «alliance des élites » (jingying lianmeng) et «les masses paysannes et ouvrières » (nong gong dazhong). Assurément, le contenu de ces débats s'inscrit dans une continuité historique.

Je souhaite maintenant évoquer le travail d'un autre professeur américain, Franz Schurmann qui, très tôt, en 1966, publia un ouvrage intitulé Idéologie et organisation en Chine communiste ${ }^{8}$. Ce livre, bien qu'empreint d'un fonctionnalisme sociologique très marqué, analyse en profondeur les raisons pour lesquelles la Chine nouvelle s'est graduellement émancipée du système soviétique. Schurmann explique que les dirigeants du pays, en 1949, confrontés aux exigences de l'industrialisation et de la modernisation, furent confrontés à un choix: fallait-il suivre l'exemple de l'URSS ou perpétuer la «voie, proprement chinoise, de Yan'an»?

La voie soviétique se caractérise par une forte dépendance à l'égard des spécialistes pour mettre en œuvre l'économie planifiée et centralisée. La voie de Yan'an, elle, repose avant tout sur les masses et leur mobilisation. Emprunter la voie soviétique, c'est donner la préséance au technicien, à une politique qui favorise les intellectuels. Choisir celle de Yan'an, c'est d'abord privilégier les masses et le peuple, leur «esprit d'initiative». Dès lors, Mao, dans les années 1950, insiste sur la nécessité de résoudre les contradictions entre «le rouge et l'expert», sur l'importance des relations entre "politique et compétence professionnelle». Pour Schurmann, tous ces éléments sont à relier à des structures fondamentales et à la stratification de la société chinoise. [...]

Analysant le choix de Mao de saper tout effort pour instaurer une planification centralisée, Schurmann explique qu'imiter le modèle soviétique revenait à l'époque à s'exposer à de lourdes conséquence politiques. C'était en effet s'en remettre à un petit nombre de départements et de technocrates alors même que la base sociale du Parti était composée de paysans et d'ouvriers. Cette base risquait donc d'être marginalisée et de se voir nier tout rôle dans l'entreprise d'industrialisation et de modernisation du parti. Un certain nombre de documents rendus publics ces dernières années confirment cette analyse. Dès lors, le Grand bond en avant incita au contraire chaque cadre à s'engager dans l'effort économique et les masses à participer à la modernisation du pays. Il s'agit là d'une phase de l'histoire que nous connaissons bien, mais que nous imputons souvent à un coup de folie de Mao. Or, l'analyse de Schurmann est plus subtile. Le Grand bond en avant, le pouvoir donné à la base (provinces, localités, comtés), les slogans de «soumission de l'expert au profane» ou de «direction de l'économique par le politique», etc., tout cela est intimement lié aux structures

8. Schurmann Franz, Ideology and Organization in Communist China, Berkeley, University of California Press, 1968. 
politiques de la société chinoise de l'époque. En d'autres termes, ce que Mao exigeait de la base sociale du Parti, c'était qu'elle devienne le sujet et maître de la modernisation du pays ; ce qu'il redoutait, c'était qu'elle se fasse marginaliser par la bureaucratie et les technocrates.

À partir du Grand bond en avant, on quitte donc le modèle soviétique pour retrouver «la voie de Yan'an», celle de la mobilisation des masses et des localités. Pour beaucoup, aujourd'hui, une telle voie n'est pas pertinente et le seul impératif est de promouvoir le savoir, la science, la technique, la culture etc. En quoi serait-il utile de se reposer sur les ouvriers et les paysans? Mais, en réalité, une telle position reflète une vision très partielle des choses, qui souffre d'une méconnaissance des fondements de la science politique. Le destin politique d'un parti de gouvernement aujourd'hui dépend de l'existence d'une grassroots base (caogen jichu) qui lui apporte son soutien : ce n'est pas autre chose que ce qu'on a appelé en Chine le «soutien des masses» (qunzhong jichu). Si un parti est d'abord constitué d'un groupe de prix Nobel, non seulement il est complètement inutile, mais il se méprend de surcroît profondément sur ce qu'est le politique. Pour n'importe quel parti de gouvernement dans un pays occidental, cela relève simplement du sens commun. Tout parti dépend pour vivre de sa base populaire. Alors que Mao, autour de 1969, augmentait la représentation des ouvriers et des paysans au comité central, les partis démocrate et républicain faisaient aux États-Unis des réformes du même ordre ayant pour objectif d'acquérir une meilleure représentativité en tant que partis de gouvernement. Le Parti démocrate américain en 1968 a d'abord déterminé de manière rigide la proportion de noirs, de latinos ou de femmes qui devaient figurer parmi ses représentants, et le Parti républicain a été forcé de suivre puisque tous deux se disputaient le soutien des masses. Au cœur de la Révolution culturelle, Mao demandait également que, parmi les membres du comité central, figurent des proportions de paysans, d'ouvriers ou de femmes. Il insistait sur la base sociale, voulait que la structure de la société se retrouve dans les hautes instances du Parti. Aujourd'hui, on accorde une importance excessive aux curriculum vitae, aux diplômes, ce qui aboutit à voir les choses de manière partielle. Or, s'il n'y a pas de politique conduite à la base, s'il n'y a pas de base populaire, s'il n'y a que des technocrates qui s'activent avec autorité, cela ne conduit pas à grand chose!

\section{Conclusion}

Il y a un peu plus de dix ans, dans un essai intitulé «Le libéralisme est-il fait pour des aristocrates ou pour le peuple?», j'avançais, avec autant de force que je le pouvais, les propositions suivantes : «Aujourd'hui, les grands discours 
Prendre en compte la continuité historique pour penser le politique aujourd'hui

des intellectuels chinois sur le libéralisme n'évoquent que leur propre liberté ou celle des patrons. C'est la liberté des riches, des forts, de ceux qui sont capables d'agir! Mais parallèlement, nul ne dit mot sur le fait que, pour le libéralisme, le point de départ de la théorie des droits, c'est que ceux-ci appartiennent à tous, et notamment à ceux qui sont impuissants à les défendre: les faibles, les infortunés, les pauvres, les prolétaires, les ignorants...». Je formulais à l'époque mes doutes de la façon suivante: "Que font en réalité les intellectuels chinois ? Mettent-ils le pouvoir qu'ils tirent de leur savoir au service d'une minorité jouissant de droits spéciaux ou promeuvent-ils les droits de tous?». J'en étais venu à penser qu'un certain nombre de ceux que l'on décrit comme des «intellectuels » étaient en fait devenus des fonctionnaires ou s'étaient transformés en «serviteurs des riches».

Je me souviens aussi qu'à l'époque, quand cet article fut publié, il suscita des réactions assez fortes dans le monde intellectuel. Parmi même mes vieux amis, beaucoup trouvaient irrecevables mes propos, estimant que ma position était bien trop unilatérale et, de plus, complètement déconnectée tant de la réalité des réformes que de celle du monde académique. Or, les critiques féroces à l'égard des élites intellectuelles que l'on peut lire sur les médias et les sites Internet les plus divers sont désormais quotidiennes et ce, sur un ton plus véhément encore que le mien à l'époque. À partir d'environ 2002, les intellectuels de gauche comme de droite se sont tous mis à évoquer une «alliance des élites » (jingying lianmeng), persuadés qu'en Chine aujourd'hui, les élites politiques, économiques et intellectuelles s'étaient déjà liguées par intérêt commun afin de former une alliance relativement stable contrôlant le pays (tongzhi jingying lianmeng) en écartant les individus des classes plus modestes. En reprenant la formule de Wen Jiejun ${ }^{9}$, on peut dire qu'on aboutit à une union des puissants, «les hauts fonctionnaires, ceux qui contrôlent l'appareil de production, les intellectuels et les médias», et que cette union exclut les ouvriers, les paysans, et les groupes sociaux les plus faibles (ruoshi qunti). En tout état de cause, à gauche comme à droite, chacun souligne désormais que c'est la relation entre les élites et les masses qui est aujourd'hui devenue la contradiction la plus aiguë dans la société chinoise. Si l'on garde à l'esprit ce qui a été précédemment évoqué, on réalise qu'une telle contradiction pose en réalité un problème fondamental, qui existe déjà depuis les débuts de la période maoïste. En ce sens, il existe aussi une continuité des problèmes à travers ces soixante années de la République.

Mon analyse de la situation actuelle va quelque peu à l'encontre du discours dominant. Si l'on se réfère à la situation de ces dernières années, on ne

9. Wen Jiejun (1950 -), célèbre économiste professeur à l'Université du peuple de Pékin et spécialiste de la question des campagnes. N.d.T. 
peut conclure de manière simple qu'une alliance irrésistible des élites s'est déjà constituée, et qu'elle s'effectue dans un geste conscient d'exclusion des masses. Les faits, au contraire, plaident pour une autre analyse: en réalité, les intellectuels, les médias ou les fonctionnaires ont tous évolué pendant la période récente. Que l'on prenne d'abord l'exemple du monde académique. Il me semble qu'en son sein, ceux qui s'identifient consciemment aux puissants, s'érigeant en défenseurs de leur intérêt, ne forment en fait qu'une minorité. Même si celle-ci est puissante, car constituée d'intellectuels qui occupent aussi des positions de pouvoir dans l'administration (xueguan), la plupart des professeurs et chercheurs compatit malgré tout aujourd'hui au sort des masses et des moins nantis. Si l'on évoque ensuite les médias, force est de constater à quel point ils ont également évolué ces dernières années. On ne pourra que noter le rôle utile qu'ils ont pu jouer en se souciant des plus modestes. Parallèlement, je crois qu'il n'y a pas lieu de diaboliser les fonctionnaires - et notamment les cadres locaux - pour ne porter qu'un regard négatif sur la situation, car on ne leur rend pas ainsi justice. En réalité, bien des cadres locaux sont proches des masses et des plus faibles ${ }^{10}$.

Il me semble donc que, depuis quelques années, le «nouveau consensus sur les réformes » décrit plus haut est en train de se former et que ce consensus jouit d'une base sociale relativement large. Il ne rassemble pas simplement les paysans, les ouvriers et les classes modestes, mais aussi des intellectuels, des fonctionnaires et des travailleurs des médias. De ce fait, il a une influence réelle sur l'orientation des réformes et sur bon nombre de politiques concrètes.

10. Gan Yang va ici à l'encontre d'un discours très répandu qui, face aux multiples problèmes sociaux rencontrés en Chine, pointe d'abord la responsabilité de cadres locaux peu soucieux du peuple et corrompus. (Notons au passage que ce discours a lui-même plusieurs strates : s'il fait évidemment souvent référence à des réalités objectives, il peut aussi être facilement employé pour limiter la responsabilité des échelons hiérarchiques supérieurs de la bureaucratie en désignant des boucs émissaires. Ultimement, il peut même renforcer la probité et donc la légitimité d'un leadership central quand celui-ci intervient, dans des situations concrètes, pour punir les coupables). N.d.T. 
Prendre en compte la continuité historique pour penser le politique aujourd'hui

\title{
GLOSSAIRE
}

\author{
caogen jichu 草根基礎 \\ di wo maodun 敵我矛盾 \\ Gongyang 公羊 \\ guoqing 國情 \\ hong yu zhuan 紅與專 \\ jingying lianmeng 精英聯盟 \\ jiu gaige gongshi 舊改革共識 \\ nong gong dazhong 農工大眾 \\ qunzhong tichu 群眾基礎 \\ renmin neibu maodun 人民內部矛盾 \\ renqing 人情 \\ rujia shehuizhuyi gongheguo 儒家社會主義共和國 \\ ruoshi qunti 弱勢群體 \\ tong san tong 通三統 \\ xiangqing 鄉情 \\ xiang zhen qiye 鄉鎮企業 \\ xueguan 學官 \\ $\mathrm{xu}$ jun gonghe 虛君共和 \\ yi ren wei ben 以人為本 \\ Zhongguo daolu 中國道路 \\ Zhonghua 中華 \\ Zhonghua renmin gongheguo 中華人民共和國
}

